\title{
Continuum Breakdown Parameter Based on Entropy Generation Rates
}

\author{
José A. Camberos ${ }^{\dagger}$ \\ United States Air Force Research Laboratory \\ Wright-Patterson AFB, OH 45433 \\ Po-Heng Chen* \\ Department of Aerospace Engineering, University of Michigan \\ Ann Arbor, MI 48109
}

\begin{abstract}
The dimensionless ratio of the mean free path to a reference length scale typically quantifies the extent to which the continuum assumption in fluid flow dynamics breaks down. However, ambiguity exists in the choice of an appropriate length scale and some researchers have suggested a local parameter, like the Knudsen number, for quantifying continuum breakdown. The authors review a few selected parameters and suggest a continuum breakdown parameter based on local entropy production rates. The selected parameter was chosen by observing and comparing alternatives and abstracting common elements and results. Results presented include laminar boundary flow and one-dimensional shock wave structure. Although both cases are well within the continuum description, they provide an ideal setting for comparison since both have semi-analytical solutions.
\end{abstract}

\section{INTRODUCTION}

Interest in rarefied gas dynamics continues to be stimulated by the possibility of high-speed flight at very high altitudes. Rarefied gas dynamics describes flow in which the length of the molecular mean free path is comparable to some macroscopic reference dimension of the flow field. Under these conditions the gas does not behave entirely as a continuous fluid but rather exhibits some of the properties of its coarse molecular structure. Rarefied gas effects include regions of sharp gradients in velocity, pressure, and/or temperature. In some cases, one or more flow regimes, including free molecule, transition, slip, and continuum flow, may be important. Because of the different physical processes involved in each flow regime, different mathematical models must be used, each of which requires alternative numerical techniques for computational simulation (e.g., Direct Simulation Monte Carlo versus Computational Fluid Dynamics). The dimensionless ratio of the mean free path to a reference length scale typically quantifies the extent to which the continuum assumption breaks down. However, ambiguity exists in the choice of an appropriate length scale and some researchers have suggested a local parameter, like the Knudsen number, for quantifying continuum breakdown (Bird, [1], [2]). In fact, as discussed in Chapter 1 of Bird [2], the limitations of the conservation equations (Navier-Stokes) appears to be directly related to a mathematical requirement for determinate set of equations. The physical processes affecting these relations are classified as nonequilibrium effects and not limited to rarefied gas dynamics. The transport terms like shear stresses and heat fluxes do not form a closed set when gradients in the macroscopic variables become so steep that their scale length is of the same order as the molecular mean-free path. The Knudsen number

$$
K n=\lambda / L
$$

\footnotetext{
${ }^{\dagger}$ Aerospace Engineer and AIAA Senior Member.

* Graduate Student, Summer 2002 Research Associate at WPAFB, AIAA Student Member (chenph@umich. edu).
} 
quantifies the degree to which this condition is met. The traditional requirement stipulates that the Navier-Stokes equations are valid up to a Knudsen number of 0.1. A more precise limit specifies a local Knudsen number where the characteristic length may be replaced by the local length scale of the macroscopic gradients. The macroscopic gradients may be density, velocity, temperature, or pressure and variations of these have appeared in the literature (see Wang \& Boyd [4] and Wang, Sun, Boyd [5] for more detail). The accuracy of the governing Navier-Stokes equations begins to degrade around a Knudsen number of 0.1 while a suggested upper limit for the continuum model may be taken as 0.2 (Bird [2], pp. 2). Transitional flow is often categorized as falling between a Knudsen-number value of 0.01 to 0.1 , although these values are not universally accepted. Two other dimensionless parameters that depend on local flow gradients provide some measure of continuum breakdown. These were discussed by P. Canupp [3] and defined by:

$$
K_{\tau} \equiv \frac{|\tau|}{p}, \quad K_{q} \equiv \frac{|q|}{p c} .
$$

Here we review these few selected parameters and suggest a continuum breakdown parameter based on local entropy production rates. This approach was inspired by the work presented in Wang and Boyd [4], where this particular issue was raised. First, we review the entropy balance equations from the continuum standpoint, based on the extension of the second law of thermodynamics for unsteady fluid flow. Second, we propose to define a continuum breakdown parameter based on a dimensionless formula for the entropy generation rate. Third, we present a comparison for a few selected cases between breakdown parameters. Further work is underway utilizing full CFD solutions of the Navier-Stokes equations and will be presented in a follow-on paper. The focus of the present paper is on developing the appropriate formulas for calculation and comparison.

\section{Entropy Balance Equations}

The governing equations of fluid dynamics can be written in compact notation as

$$
\frac{\partial \boldsymbol{Q}}{\partial t}+\frac{\partial \boldsymbol{f}_{1}}{\partial x_{1}}+\frac{\partial \boldsymbol{f}_{2}}{\partial x_{2}}+\frac{\partial \boldsymbol{f}_{3}}{\partial x_{3}}=0
$$

where $\boldsymbol{Q}$ represents the state vector and $\boldsymbol{f}_{j}$ the algebraic flux vector of state quantities. These can represent either the Euler or Navier-Stokes equations. For an ideal gas the entropy formula is readily available from thermodynamics, such that $S=S(\boldsymbol{Q})$ with respect to the state variables

$$
\boldsymbol{Q}=(\rho, \rho u, \rho v, \rho w, \rho e) .
$$

The specific entropy formula, from thermodynamics, is

$$
s(\rho, T)-s_{0}=c_{V} \ln \left(T / T_{0}\right)-R \ln \left(\rho / \rho_{0}\right) .
$$

The total specific energy is

$$
\rho e=\frac{1}{2} \rho\left(u^{2}+v^{2}+w^{2}\right)+\frac{1}{\gamma-1} \rho R T,
$$

so that the temperature can be written as

$$
R T(\boldsymbol{Q})=(\gamma-1)\left\{\frac{q_{5}}{q_{1}}-\frac{1}{2}\left(\frac{q_{2}^{2}+q_{3}^{2}+q_{4}^{2}}{q_{1}^{2}}\right)\right\} .
$$

Setting the constant reference state $s_{0}$ equal to zero and writing $T$ in terms of the state variables gives

$$
S(\boldsymbol{Q})=\rho s=\frac{q_{1}}{\gamma-1} \ln \left\{\frac{(\gamma-1)}{2}\left[\frac{2 q_{1} q_{5}-\left(q_{2}^{2}+q_{3}^{2}+q_{4}^{2}\right)}{q_{1}^{\gamma+1}}\right]\right\}
$$


where the gas constant provides a convenient way to render the entropy dimensionless. The balance of entropy emerges by substituting (1) into the expression:

$$
\frac{\partial S}{\partial t}+\frac{\partial S}{\partial \boldsymbol{Q}} \cdot \frac{\partial \boldsymbol{Q}}{\partial t}=0 \quad \rightarrow \quad \frac{\partial S}{\partial t}-\sum_{j=1}^{3} \frac{\partial S}{\partial \boldsymbol{Q}} \frac{\partial \boldsymbol{f}_{j}}{\partial x_{j}}=0
$$

If the fluxes contain only the convective transport of mass, momentum, and energy as represented in the Euler equations, then it is possible to show that

$$
\sum_{j=1}^{3} \frac{\partial S}{\partial \boldsymbol{Q}} \frac{\partial \boldsymbol{f}_{j}}{\partial x_{j}}=\sum_{j=1}^{3} \frac{\partial F_{j}}{\partial x_{j}}
$$

where the entropy fluxes are $F_{j}=\rho s u_{j}$ and the entropy generation rate

$$
\dot{S}_{\text {gen }}=\frac{\partial S}{\partial t}+\sum_{j=1}^{3} \frac{\partial F_{j}}{\partial x_{j}}=0
$$

demonstrates that entropy generation is zero for adiabatic, inviscid (reversible) gas dynamics. If the fluxes contain terms that model viscosity and heat conduction, then

$$
\frac{\partial S}{\partial \boldsymbol{Q}} \frac{\partial \boldsymbol{f}_{j}}{\partial x_{j}}=\frac{\partial F_{j}}{\partial x_{j}}+\frac{\partial}{\partial x_{j}}\left(\frac{q_{j}}{T}\right)-\frac{\tau_{j i}}{T} \frac{\partial u_{i}}{\partial x_{j}}+\frac{q_{j}}{T^{2}} \frac{\partial T}{\partial x_{j}}
$$

where $q_{j}$ represents the heating rate per unit area (heat flux) and $\tau_{j i}$ the viscous stress tensor. On substitution in the entropy balance equation, we get two expressions that represent entropy generation:

$$
\begin{aligned}
& \dot{S}_{\mathrm{gen}}=\frac{\partial S}{\partial t}+\frac{\partial F_{j}}{\partial x_{j}}+\frac{\partial}{\partial x_{j}}\left(\frac{q_{j}}{T}\right) \\
& \dot{S}_{\mathrm{gen}}=\frac{\tau_{j i}}{T} \frac{\partial u_{i}}{\partial x_{j}}-\frac{q_{j}}{T^{2}} \frac{\partial T}{\partial x_{j}} .
\end{aligned}
$$

We identify the second expression by collecting all terms that have a definite sign. Equation (13.a) may be obtained by generalizing the second law of thermodynamics for an open, unsteady system. One can derive (13.b) directly from (13.a) by using all the constitutive relations and conservation equations. The procedure is tedious and not straight-forward. The procedure outlined above is atypical but offers a methodological advantage (Camberos, [6]).

We propose a continuum breakdown parameter based on entropy generation rate defined by the dimensionless parameter:

$$
K_{\mathrm{S}} \equiv \frac{\ell \dot{S}_{\mathrm{gen}}}{\rho R \sqrt{R T}}
$$

Comparing this expression with Equation (2), we see that the entropy generation rate in dimensionless form includes portions of both $K_{\tau}$ and $K_{q}$ and therefore represents the effects of both velocity and temperature gradients. In particular, it is hoped that this expression may serve at least as a theoretical benchmark for quantifying the effects of that herald the onset of continuum breakdown. We consider in the sections that follow two examples to demonstrate the concept for problems that have a theoretical or nearly-analytic solution. Regions of interest in the general simulation of fluid flow include boundary layers and shock waves where the transport properties are most significant. Both regions contain entropy generation due to viscous dissipation and heating. Therefore, one would expect these regions to serve as ideal candidates for comparing breakdown parameters. The accepted criterion is to declare the onset of continuum breakdown when the value of the relevant parameter equals 0.1 . With both the boundary layer and shock profile, we will explore and compare the various parameters keeping this value in mind. 


\section{EXAMPLE 1: INCOMPRESSIBLE BOUNDARY LAYER}

Neglecting buoyancy, the steady 2-D incompressible flow (boundary layer) equations are:

$$
\begin{array}{ll}
\text { Mass: } & \frac{\partial u}{\partial x}+\frac{\partial u}{\partial y}=0 \\
x \text {-Momentum: } & u \frac{\partial u}{\partial x}+v \frac{\partial u}{\partial y}=U \frac{\partial u}{\partial x}+v \frac{\partial^{2} u}{\partial y^{2}}
\end{array}
$$

with the boundary conditions $u(x, 0)=v(x, 0)=0$ and $u(x, y \rightarrow \infty)=U(x)$. Conditions in the free-stream and outside the boundary layer are denoted by the $\infty$ symbol.

\section{Blasius' Equation}

L. Prandtl conceived the concept of a boundary layer and it was his first student, H. Blasius who introduced similarity analysis and assumed small displacement thickness so that $U=$ const. and $d U / d x=0$ to obtain a solution. If we use the dimensionless similarity variable and stream function:

$$
\eta=y \sqrt{\frac{U}{2 v x}} \quad \psi=\sqrt{2 v U x} f(\eta) \quad v=-\frac{\partial \psi}{\partial x}=f^{\prime} \frac{y U}{2 x}-\sqrt{\frac{v U}{2 x}} f
$$

This makes the velocities:

$$
u=\frac{\partial \psi}{\partial y}=U f^{\prime}(\eta) \quad v=-\frac{\partial \psi}{\partial x}=\sqrt{\frac{v U}{2 x}}\left(\eta f^{\prime}-f\right)
$$

The momentum equation reduces to the Blasius equation for a flat plate:

$$
f^{\prime \prime \prime}+f f^{\prime \prime}=0 \text {. }
$$

The boundary conditions are: $\quad \mathrm{u}(\mathrm{x}, 0)=\mathrm{v}(\mathrm{x}, 0)=0 \Rightarrow f^{\prime}(0)=f(0)=0$

and

$$
u(x, \infty)=U \quad \Rightarrow f^{\prime}(\infty)=1 .
$$

The accepted value for the $f^{\prime \prime}(0)$ found by numerical solution of (19) and iteration is 0.469600 . At $f^{\prime}=0.99 \rightarrow$ $\eta=3.5$, so that a complete solution provides a measure of the boundary layer thickness, the displacement thickness, the friction coefficient, wall shear stress and friction drag.

\section{Flat-Plate Heat Transfer for Constant Wall Temperature}

To account for the effects of temperature gradients, it is necessary to include the energy equation, even though this is decoupled from the Blasius momentum equation. Consider the case where heat transfer occurs at constant wall temperature $T_{w}$ and define the dimensionless temperature difference as $\Theta(\eta)=\left(T-T_{\infty}\right) /\left(T_{w}-T_{\infty}\right)$. Without neglecting dissipation (contrary to what is assumed in textbooks), the energy equation reduces to

$$
\Theta^{\prime \prime}+\operatorname{Pr} . f . \Theta^{\prime}+\operatorname{Pr} . \operatorname{Ec} \cdot\left(f^{\prime \prime}\right)^{2}=0 \text {, }
$$

with boundary conditions $\Theta(0)=1$ and $\Theta(y \rightarrow \infty)=0$. Pr is the Prandtl number and Ec is the Eckert number defined as: $\mathbf{E c}=U^{2} / c_{\mathrm{p}} \Delta T$, where $\Delta T$ equals the temperature difference between the fixed wall temperature and the free-stream. 


\section{Flat-Plate Laminar Boundary Layer Entropy Production}

The details presented above are duplicated from what one can find in the literature and textbooks on fluid mechanics in particular (e.g., White [7], pp. page 86-88). We repeat them here for convenience in deriving the following. The formula for entropy generation rate consistent with the boundary layer equations can be written as

$$
\dot{S}_{g e n}=\frac{\Phi}{T}-\frac{q_{j}}{T^{2}} \frac{\partial T}{\partial x_{j}} .
$$

Where $\Phi$ represents the viscous dissipation function. The complete formula for the viscous dissipation function in Cartesian coordinates, assuming a Newtonian fluid, can be reduced to 2D incompressible flow as

$$
\Phi=\tau_{i j}^{\prime} \frac{\partial u_{i}}{\partial x_{j}}=\mu\left\{2\left(\frac{\partial u}{\partial x}\right)^{2}+2\left(\frac{\partial v}{\partial y}\right)^{2}+\left(\frac{\partial u}{\partial y}+\frac{\partial v}{\partial x}\right)^{2}\right\}
$$

Fourier's law for the heat conduction (2D) gives

$$
q_{j} \frac{\partial T}{\partial x_{j}}=q_{x} \frac{\partial T}{\partial x}+q_{y} \frac{\partial T}{\partial y}=-k\left\{\left(\frac{\partial T}{\partial x}\right)^{2}+\left(\frac{\partial T}{\partial y}\right)^{2}\right\}
$$

So that the complete $2 \mathrm{D}$ incompressible entropy generation rate is represented by

$$
\dot{S}_{\mathrm{gen}}=\frac{\mu}{T}\left\{2\left(\frac{\partial u}{\partial x}\right)^{2}+2\left(\frac{\partial v}{\partial y}\right)^{2}+\left(\frac{\partial u}{\partial y}+\frac{\partial v}{\partial x}\right)^{2}\right\}+\frac{k}{T^{2}}\left\{\left(\frac{\partial T}{\partial x}\right)^{2}+\left(\frac{\partial T}{\partial y}\right)^{2}\right\} .
$$

Utilizing the standard similarity transformation, we now fill in the derivatives using the similarity functions and parameters:

$$
\begin{aligned}
& \frac{\partial u}{\partial x}=U f^{\prime \prime}(\eta) \frac{\partial \eta}{\partial x}, \quad \frac{\partial u}{\partial y}=U f^{\prime \prime}(\eta) \frac{\partial \eta}{\partial y}, \quad \frac{\partial v}{\partial x}=-\frac{1}{2 x} \sqrt{\frac{\nu U}{2 x}}\left(\eta f^{\prime}-f\right)+\sqrt{\frac{\nu U}{2 x}} \eta f^{\prime \prime} \frac{\partial \eta}{\partial x} \\
& \frac{\partial T}{\partial x}=\left(T_{w}-T_{e}\right) \Theta^{\prime}(\eta) \frac{\partial \eta}{\partial x}, \quad \frac{\partial T}{\partial y}=\left(T_{w}-T_{e}\right) \Theta^{\prime}(\eta) \frac{\partial \eta}{\partial y}, \quad \frac{\partial v}{\partial y}=\sqrt{\frac{v U}{2 x}} \eta f^{\prime \prime} \frac{\partial \eta}{\partial y} .
\end{aligned}
$$

From the definition of $\eta$, we can substitute and simplify to obtain the following, where an order-of-magnitude inspection of terms gives the entropy generation rate:

$$
\dot{S}_{\mathrm{gen}} \cong \frac{\mu}{T}\left(\frac{\partial u}{\partial y}\right)^{2}+\frac{k}{T^{2}}\left(\frac{\partial T}{\partial y}\right)^{2}=\frac{\rho U^{3}\left(f^{\prime \prime}\right)^{2}}{2 T x}+\frac{\rho U c_{p}\left(T_{w}-T_{e}\right)^{2}\left(\Theta^{\prime}\right)^{2}}{2 \operatorname{Pr} T^{2} x}
$$

where $\operatorname{Pr}=\mu \mathrm{c}_{\mathrm{p}} / \kappa$ (Prandtl number). Several possibilities exist for making the expression dimensionless. Since entropy is most closely associated with thermodynamic effects, we can choose to divide by the thermal energy cluster $\rho U c_{p} / x$ to obtain

$$
\dot{S}_{g e n}^{*} \equiv \frac{x \dot{S}_{g e n}}{\rho U c_{p}}=\frac{U^{2}}{2 c_{p} T_{e}}\left(f^{\prime \prime}\right)^{2}\left(1+\frac{\Delta T}{T_{e}} \Theta\right)^{-1}+\frac{\left(\Theta^{\prime}\right)^{2}}{2 \operatorname{Pr}}\left(\frac{T_{w}}{T_{e}}-1\right)^{2}\left(1+\frac{\Delta T}{T_{e}} \Theta\right)^{-2} .
$$


However, it is typically more convenient to express dimensionless quantities in terms of more familiar and easily specified parameters like Mach and Reynolds numbers. Using the ideal gas relations given by

$$
c_{p}=\frac{\gamma R}{\gamma-1}, \quad U_{\infty}=M_{\infty} \sqrt{\gamma R T_{\infty}}, \quad \operatorname{Re}_{\ell}=\frac{\rho_{\infty} U_{\infty} \ell}{\mu_{\infty}},
$$

we rewrite the Knudsen-like parameters as follows.

$$
\begin{aligned}
& \text { Entropy: } \quad K_{S}=\frac{\ell}{x}\left\{\frac{M_{\infty}^{3}}{2} \gamma \sqrt{\gamma}\left(f^{\prime \prime}\right)^{2}\left(1+\frac{\Delta T}{T_{\infty}} \Theta\right)^{-1}+\frac{\left(\Theta^{\prime}\right)^{2}}{2 \operatorname{Pr}} \frac{M_{\infty} \gamma \sqrt{\gamma}}{(\gamma-1)}\left(\frac{\Delta T}{T_{\infty}}\right)^{2}\left(1+\frac{\Delta T}{T_{e}} \Theta\right)^{-2}\right\} \\
& \text { Knudsen: } \quad K n=\frac{\lambda}{\ell}=\frac{\mu}{\ell \rho} \sqrt{\frac{\pi}{2 R T_{\infty}}}=\sqrt{\frac{\gamma \pi}{2}} \frac{M_{\infty}}{\operatorname{Re}_{\ell}} \\
& \text { Viscous Stress: } \quad K_{\tau}=\frac{|\tau|}{p}=\frac{\mu}{\rho R T}\left|\frac{\partial u}{\partial y}\right|=\frac{\mu U_{\infty}}{\rho R T} \sqrt{\frac{\rho U_{\infty}}{2 \mu x}}\left|f^{\prime \prime}(\eta)\right|=\frac{\gamma M_{\infty}^{2}}{\operatorname{Re}_{\ell}} \sqrt{\frac{\operatorname{Re}_{\ell}}{2(x / \ell)}}\left|f^{\prime \prime}(\eta)\right| \\
& \text { Heating: } \quad K_{q}=\frac{|q|}{p c}=\frac{\Delta T}{T_{\infty}} \frac{\gamma M_{\infty}}{\operatorname{Pr}(\gamma-1) \operatorname{Re}_{\ell}} \sqrt{\frac{\operatorname{Re}_{\ell}}{2(x / \ell)}}\left|\Theta^{\prime}\right|
\end{aligned}
$$

\section{Results for Flat-Plate Boundary Layer Flow}

It is a simple matter these days with sophisticated software available to obtain a numerical solution for ordinary differential equations. Solutions for the Blasius momentum equation and the energy equation thus provide the numerical values for evaluating and comparing the various parameters. Because of the way the Knudsen-like parameters are scaled, the ratio of $(x / L)$ appears in the denominator. Therefore, near the boundary layer edge, where CFD typically requires grid clustering to solve the boundary layer equations, gradients are much more severe. In the calculations presented, we used $x=L=1.0$ to focus on the differences between the parameters at length scales comparable to the macroscopic scale. A reasonable application would be to use these parameters to detect how close to the boundary layer leading-edge a continuum method would be valid. However, it is well known that for laminar boundary layer flows, grid convergence often solves the problem long before the continuum assumption comes into question. The Blasius and energy boundary layer equations were solved with the Mathematica symbolic calculation software. Figure 1 shows the calculated solution for the velocity and temperature profiles as well as their gradients. It is evident that indeed the velocity gradient is large while the temperature gradient is not as great. Free-stream conditions were set at Mach 0.2 and standard sea-level for pressure, temperature, and density. Figure 2 shows that the main entropy-generating mechanism is due to heat transfer in the normal direction. The minute contribution due to viscous stresses validates the "small Eckert number" approximation typically assumed. The Knudsen parameters are shown in the second half of Figure 2 and obviously indicate that this problem is well within the continuum domain (the mean-free path under these conditions is of the order of $10^{-8}$ ). Entropy generation (Figure 3) is two orders of magnitude larger than even a local Knudsen number with length scale based on the velocity gradient:

$$
K_{u}=\frac{\lambda}{u} \frac{\partial u}{\partial x} \quad \text { in Figure } 2 \text { (right-hand side). }
$$

This parameter was introduced to accommodate the presence of large velocity gradients. Since entropy-based parameter scales to $1 / x$ as compared to $1 / \sqrt{x}$ for the other parameters, it is expected that it would herald the onset of continuum breakdown much sooner if leading-edge conditions were approached. 

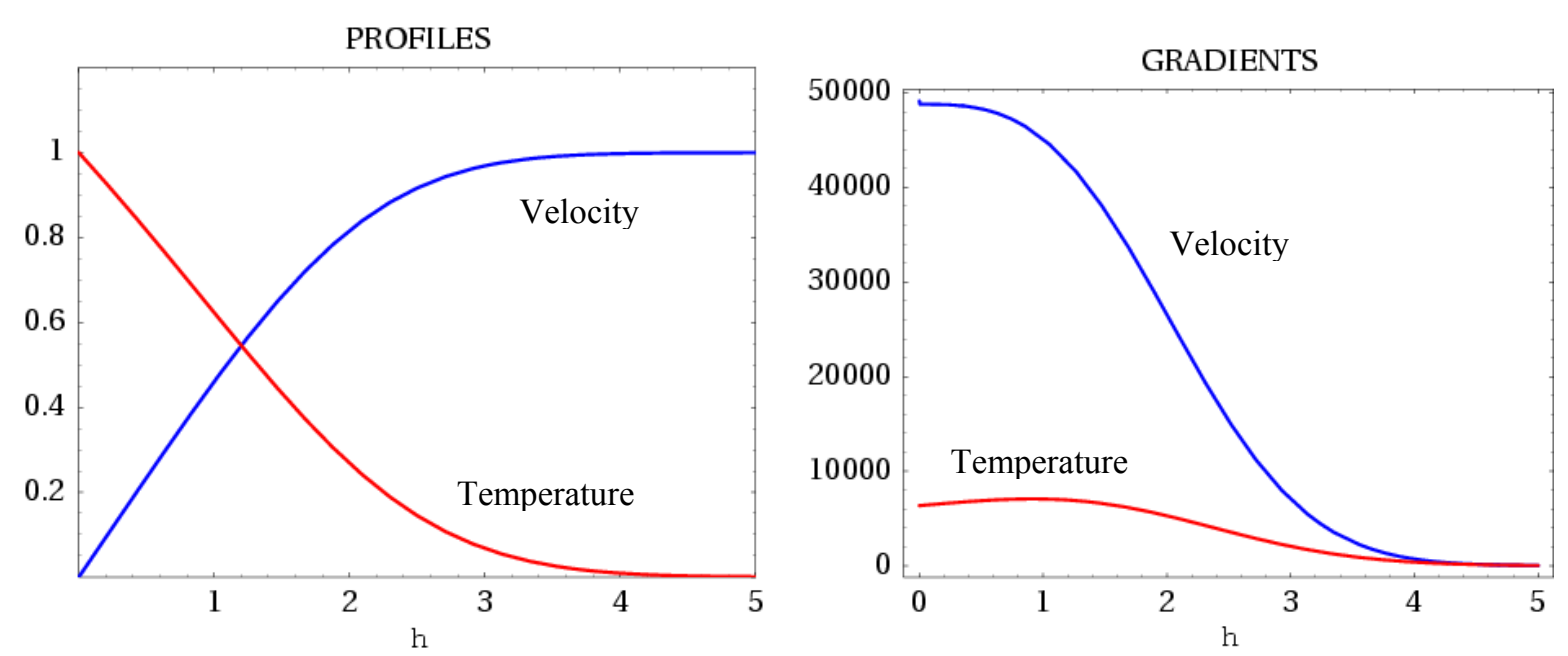

FIGURE 1. Velocity profile and temperature profiles generated with numerical ODE solution of Blasius' equation for incompressible boundary layer flow.
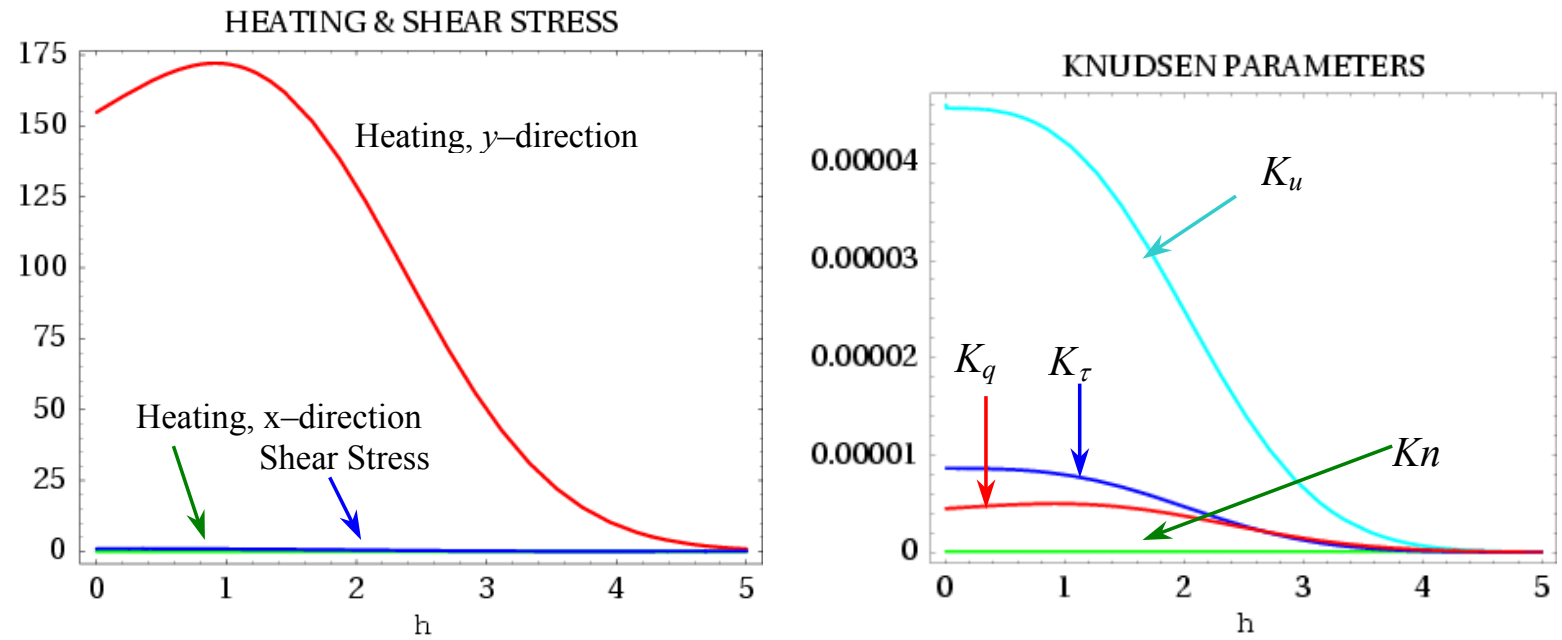

FIGURE 2. Heating and shear stress profiles (left) and Knudsen parameter profiles resulting from a numerical ODE solution of Blasius' equation for incompressible boundary layer flow.

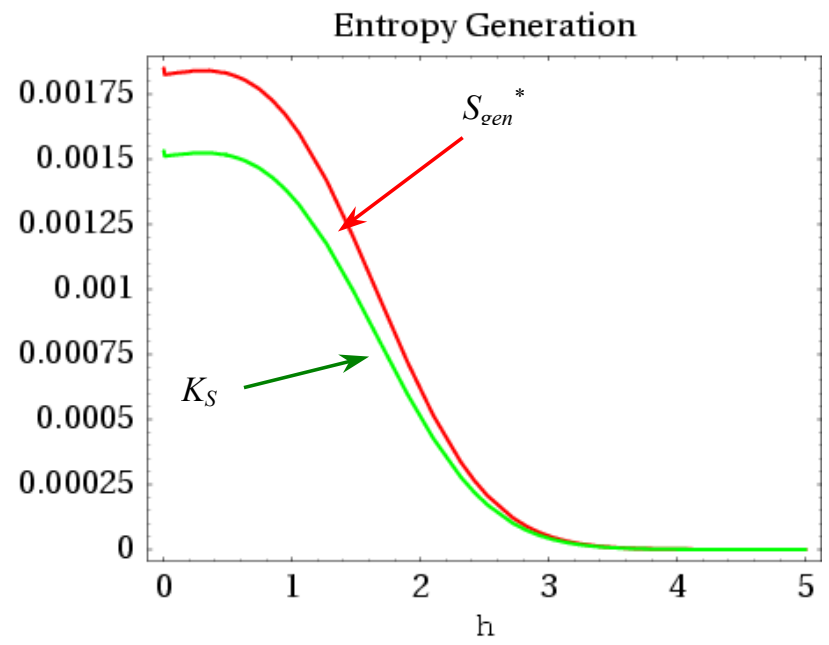

FIGURE 3. Entropy-Based Knudsen-like parameter profiles resulting from a numerical ODE solution of Blasius' equation for incompressible boundary layer flow. 


\section{EXAMPLE 2: ONE-DIMENSIONAL PLANE SHOCK WAVE}

Shock waves represent another situation where the transport properties and the closure conditions become important. For simplicity, we consider only quasi-one-dimensional flow governed by the steady Navier-Stokes equations:

$$
\begin{aligned}
& \text { Mass: } & \frac{d}{d x}(\rho u) & =0, \\
& \text { Momentum: } & \frac{d}{d x}\left(\rho u^{2}+p-\tau\right) & =0, \\
& \text { Energy: } & \frac{d}{d x}(\rho u e+p u-\tau u+q) & =0 .
\end{aligned}
$$

A unique solution exists for these equations provided that the second law of thermodynamics is satisfied. This solution represents a plane shock wave with flow direction determined by compression. These solutions have long been studied in discussing the validity of the continuum assumption (Gilbarg and Paolucci [8]). Interest continues to this day because they offer an ideal case for comparing continuum and molecular-based models relevant to the closure conditions for the transport properties. The constitutive relations for the transport properties applicable in the continuum regime are:

$$
\tau=\frac{4}{3} \mu \frac{d u}{d x}, \quad q=-\kappa \frac{d T}{d x} .
$$

The two expressions for the entropy balance equations become redundant for the steady state and are in fact interchangeable. It is therefore only necessary to calculate only one or the other:

$$
\dot{S}_{\mathrm{gen}}^{\mathrm{t}}=\frac{d}{d x}(\rho u s)+\frac{d}{d x}\left(\frac{q}{T}\right) \quad \Leftrightarrow \quad \dot{S}_{\mathrm{gen}}^{\mathrm{c}}=\frac{4}{3} \frac{\mu}{T}\left(\frac{d u}{d x}\right)^{2}+\frac{k}{T^{2}}\left(\frac{d T}{d x}\right)^{2}
$$

In unsteady situations, the two expressions should yield the same value in principle but may differ when approximate and complex numerical calculation is involved (as in sophisticated CFD algorithms).

With the right software available, it is just as easy to obtain a numerical solution to a second or higher order ordinary differential equation. However, a convenient strategy is to solve the entire problem as a system of coupled first-order ODEs. By using the equations of state for an ideal gas

$$
p=\rho R T, \quad e=\frac{1}{2} u+\frac{\rho R T}{\gamma-1}
$$

the system of equations can be reduced to solve for the macroscopic flow variables. Entropy generation rates and the other Knudsen-like parameters are obtained from the numerical solution of the system of equations, which are made dimensionless by using a reference mean-free path as the length scale. One caveat: It is generally now known that the proper approach to obtain a numerical solution to these equations is to integrate from the down-stream to the up-stream conditions. This is because the down-stream conditions represent a nodal point in velocitytemperature space, while the up-stream conditions are a saddle point. Numerical solutions that begin up-stream will soon deviate and will most likely not approach the down-stream conditions.

In the figures that follow, the calculations were obtained using a dimensionless form of the equations with all macroscopic quantities normalized by their up-stream values, a Mach number of 2, and space normalized by the up-stream mean-free path. Downstream initial conditions for numerical calculation were obtained with the Rankine-Hugoniot relations for 1D shock waves. The equations were solved with the Mathematica symbolic calculation software. 


\section{Results for One-Dimensional Shock Wave Structure}

Solutions for the 1D Navier-Stokes equations provide the numerical values for evaluating and comparing the various parameters. In the calculations presented, all breakdown parameters utilized a local value of the mean-free path as the length scale. These were defined as in Equations (13.a) and (13.b) for the entropy, Equation (2) for $K_{\tau}$ and $K_{q}$, and Equation (1) for the local Knudsen number with reference length scale based on the mass density gradient. Figure 4 shows the calculated solution for the macroscopic variable profiles as well as the heating and shear stress profiles. The profiles are centered at sonic conditions and the scale is in mean-free paths. Peak heating occurs just upstream of the sonic point while peak shearing occurs just downstream. Figure 5 shows that the pressure gradient is larger and broader than the gradients for the other variables, making a viable parameter on which to base the length scale for a Knudsen-like parameter, although density, velocity, and temperature have been mostly utilized in prior work (Wang and Boyd, [4]). In Figure 5, right-hand side, we present the entropy and entropy generation profiles. The non-monotonic behavior for the entropy, which is theoretically correct, is evident both from its profile and its gradient across the shock wave. All the parameters are presented in Figure 6 for comparison. The parameter based on heating rates across the shock spans a broad area while the entropy generation rate span is narrow. Based on this information alone, a narrower span might indicate an advantage since one would like to use the most efficient method (CFD) as far as possible into the shock interior. Unfortunately, with stronger shocks others have shown that continuum breakdown, as measured by comparing the deviation between a CFD solution and a DSMC solution indicates that continuum breakdown may occur over a broader range than indicated by any of these parameters (Wang, Sun, and Boyd [5]). This remains an issue of interest and deeper investigation.

\section{REMARKS: ENTROPY PRODUCTION IN CFD}

Inspiration for this work began with the suggestion that perhaps entropy generation rates could be utilized to detect regions of extreme velocity and temperature gradients. We have shown that this kind of parameter compares well with previously proposed alternatives. It has the advantage of including effects of velocity and temperature gradients together as well as the temporal effects associated with strongly unsteady flow, depending on how the entropy balance equation is formulated. In addition to its possible use in continuum breakdown studies, however, the second law of thermodynamics has much more general applications (Bejan [9], Scubbia [10]). We discuss some of these applications in brief and only for the purpose of setting the stage for work to follow.

A numerical method applied to the solution of the governing equations aims at calculating the updated variables from the presently known distribution at a previous time step. The space and time integration of the balance equations can be separated so that the resulting finite-volume, semi-discrete formula

$$
\frac{d \boldsymbol{Q}_{i}}{d t}+\sum_{k=1}^{m} \frac{A_{k}}{V_{i}}(\hat{n} \cdot \overrightarrow{\boldsymbol{f}})_{k}=0
$$

where $A_{k} / V_{i}$ represents the ratio of the cell-face area to the discrete cell volume, can be integrated in time to obtain new values of the state variables. Whether implicit or explicit time integration is used, the calculation of entropy generation rates can be done a posteriori, when all the other state variables are known from the solution of the fluid flow equations. For the Navier-Stokes equations the entropy transport equation (13.a) holds and one therefore may use the discrete approximations (implicit or explicit time integration):

$$
\dot{S}_{\mathrm{gen}}=\frac{(\rho s)^{n+1}-(\rho s)^{n}}{\Delta t}+\sum_{k=1}^{m} \frac{A_{k}}{V_{i}} \hat{n} \cdot\left(\rho \vec{u} s+\frac{q_{j}}{T}\right)^{*} .
$$

An alternative expression may be obtained using numerical estimates for the velocity and temperature gradients (typically already known for higher-order methods) and solving for the entropy generation rate with Equation (13.b). A numerical method that satisfies the non-negative entropy generation principle in discrete form can be said to satisfy a local form of the second law of thermodynamics. By calculating and monitoring the magnitude and sign of the entropy generation locally, one can identify regions that violate the second law. This also allows an expanded capability for ensuring that the computational simulation satisfies physical theory, is numerically stable, and incurs a minimum of numerical error. Each of these possibilities have not been fully explored and exploited. 


\section{CONCLUSIONS}

We have presented a very basic idea and sufficient detail for the definition of a continuum breakdown parameter based on local entropy generation rates. The parameter attempts to addresses the need for detecting regions where non-equilibrium physical processes dominate so that appropriate mathematical and numerical models may be employed towards the solution of the governing equations. A breakdown parameter based on entropy generation rates has the advantage of including effects of velocity and temperature gradients together as well as the temporal effects associated with strongly unsteady flow, depending on how the entropy balance equation is formulated. Although the elementary results presented rely entirely on the continuum assumption and weak non-equilibrium, by comparing the new parameter with those previously proposed and utilized, we are able to suggest its viability as a theoretical benchmark, although its use in extreme conditions has not been proven. The focus here has been in identifying a parameter for possible use in detecting regions of extreme gradients where it may be necessary to switch to a molecular-based method while using a continuum CFD solver in smoother regions. Such hybrid solutions have been explored in the past with varying degrees of success. Any effort in this direction requires a practical and robust continuum-breakdown indicator. In order to fully evaluate the entropy generation as a viable parameter, it will be necessary to first validate CFD calculations with limiting cases like those presented here and then see how well the parameter performs under more severe conditions (like strong shocks, turbulent boundary layers, and other non-equilibrium phenomena). In addition, the finite grid cell dimension in CFD provides another locally valid reference length scale to explore.

\section{ACKNOWLEDGMENTS}

This work was sponsored by the Air Force Office of Scientific Research (AFOSR) grant 2307N32A to the Air Force Research Laboratory at Wright-Patterson Air Force Base and by grant F49620-01-1-0003 to the University of Michigan under Dr. John Schmisseur. The views and conclusions contained herein are those of the authors and should not be interpreted as necessarily representing the official policies or endorsements, either expressed or implied, of the AFOSR or the U.S. Government.

\section{REFERENCES}

1. Bird, G. A., "Breakdown of Translational and Rotational Equilibrium in Gaseous Expansions", AIAA Journal Vol. 8, No. 10, pp. 1998-2003.

2. Bird, G. A., Molecular Gas Dynamics and the Direct Simulation of Gas Flows, Oxford University Press, New York: NY, 1994.

3. Canupp, P. W., "The Influence of Magnetic Fields for Shock Waves and Hypersonic Flow", AIAA Technical Paper No. 2000-2260, Denver, CO, American Institute of Aeronautics \& Astronautics, June 2000, pp. 5.

4. Wang, W. and Boyd, I. D., "Continuum Breakdown in Hypersonic Viscous Flows", AIAA Technical Paper No. 2002-0651, Reno, NV, American Institute of Aeronautics \& Astronautics, January 2002, pp. 2.

5. Wang, W., Sun, Q., and Boyd, I. D., "Towards Development of a Hybrid DSMC-CFD Method for Simulating Hypersonic Interacting Flows", AIAA Technical Paper Number 2002-3099, St. Louis, MO, American Institute of Aeronautics \& Astronautics, June 2002.

6. Camberos, J. A. "The Production of Entropy in Relation to Numerical Error in Compressible Viscous Flow", AIAA Paper No. 2000-2333, Denver, CO, American Institute of Aeronautics \& Astronautics, June 2000.

7. White, F. M., Viscous Fluid Flow, $2^{\text {nd }}$ Ed., McGraw-Hill, Boston: MA, 1991, pp. 233-241.

8. Gilbarg, D. and Paolucci, D., "The Structure of Shock Waves in the Continuum Theory of Fluids", Journal of Rational Mechanics \& Analysis, Vol. 2, pp. 617-642, 1953.

9. Bejan, A., Entropy Generation Minimization, CRC Press, Boca Raton: FL, 1996, pp. 25-30.

10. Scubbia, E. "Calculating Entropy with CFD", Mechanical Engineering Magazine, Vol. 119, No. 10, ASME International: October 1997, pp. 86-88. 

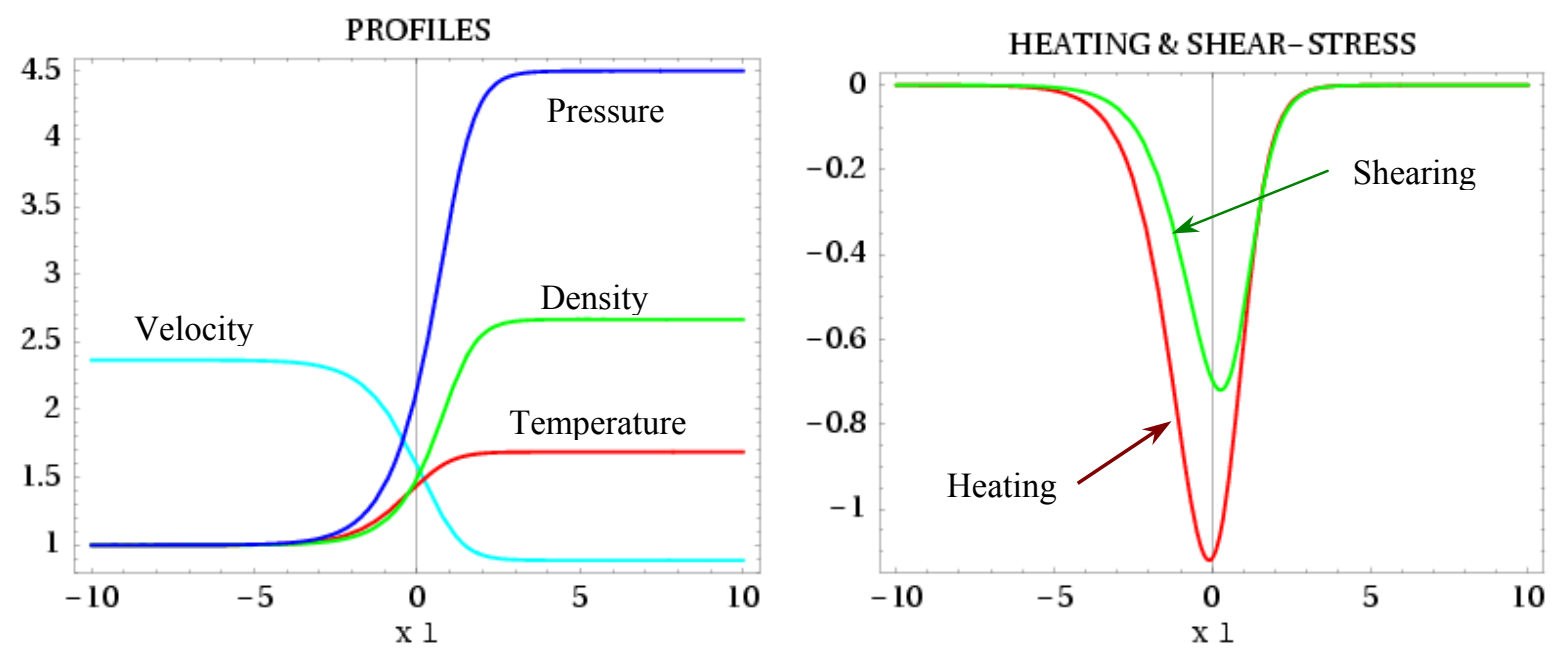

FIGURE 4. Velocity, pressure, temperature, density profiles, (left) and heating/shear stresses (right) generated with numerical ODE solution of the 1D Navier-Stokes equation for a steady normal shock.
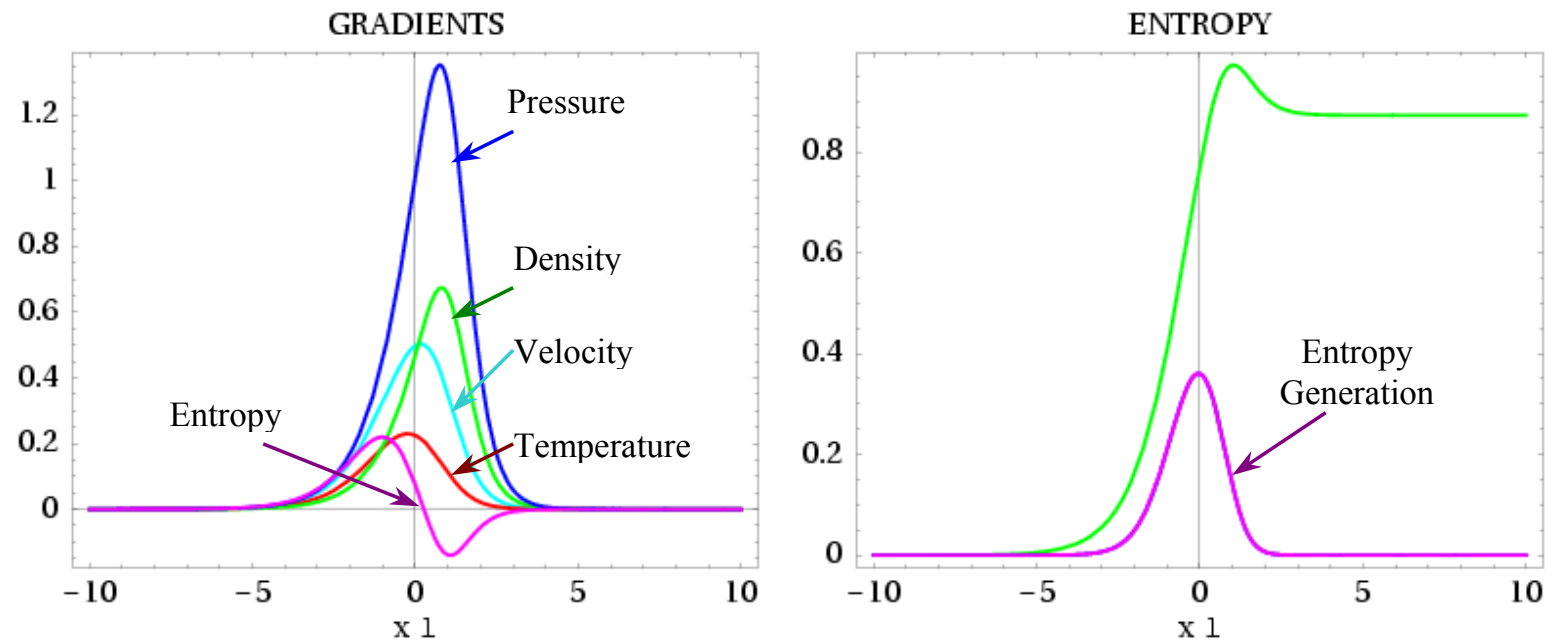

FIGURE 5. Macroscopic gradients and entropy profile profiles resulting from a numerical ODE solution for 1D shock structure.

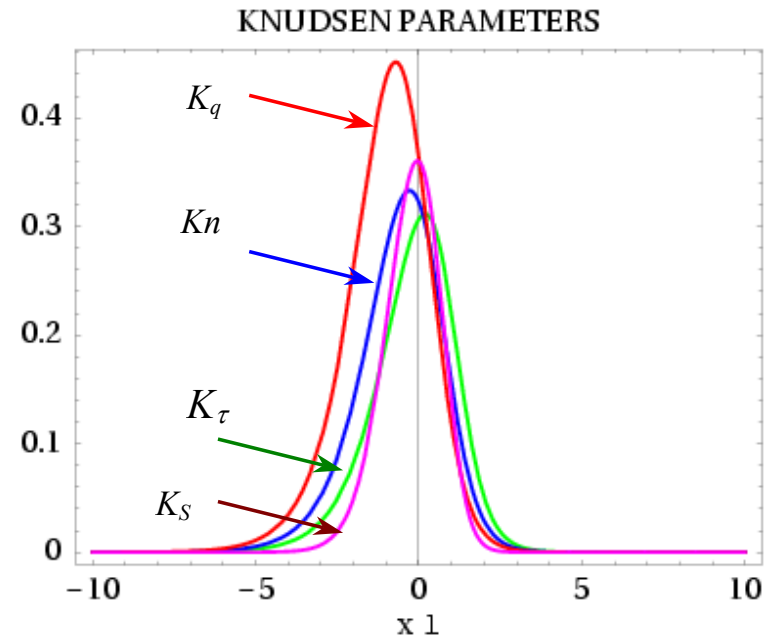

FIGURE 6. Knudsen-like parameter profiles resulting from a numerical ODE solution for normal shock wave structure. 